\title{
Impacto del tamaño de las familias afiliadas al Seguro Popular de Salud de México, experiencia 2004-2006
}

\author{
José Enrique Pérez-Salvador,Act. ${ }^{\left({ }^{(}\right)}$Cristina Gutiérrez-Delgado, D en Mat Act. ${ }^{(I)}$
}

\begin{abstract}
Pérez-Salvador JE, Gutiérrez-Delgado C. Impacto del tamaño de las familias afiliadas al Seguro Popular de Salud de México, experiencia 2004-2006. Salud Publica Mex 2010;52:234-243.
\end{abstract}

\begin{abstract}
Resumen
Objetivo. Comparar el tamaño promedio de las familias del Seguro Popular de Salud (SPS) e identificar las variables que mejor predicen la afiliación unipersonal. Material y métodos. Se comparan tamaños promedios de familias según los Padrones 2004 a 2006 del SPS y otras fuentes. Se ajustan modelos logísticos para identificar las variables explicativas de la afiliación como familia unipersonal. Resultados. Las familias del SPS en promedio tienen un miembro menos respecto de otras fuentes. Los modelos logísticos indican que cuanto más reciente es el año de afiliación tanto mayor es la probabilidad de afiliarse como familia unipersonal. Conclusiones. El menor número de miembros por familia afiliada implica un sobrefinanciamiento al SPS. Se recomienda cambiar la unidad de financiamiento hacia la persona, para apoyar el sostenimiento financiero y operativo del SPS en el mediano y largo plazos.
\end{abstract}

Palabras clave: administración en salud pública; sistemas de salud; cobertura de servicios de salud; seguridad social; México.
Pérez-Salvador JE, Gutiérrez-Delgado C. Impact of size of families in Mexico's Popular Health Insurance Program. Salud Publica Mex 2010;52:234-243.

\section{Abstract}

Objective. To compare the average size of families affiliated with Popular Health Insurance (SPS, abbrev. in Spanish) and identify variables that best predict single-person affiliation. Material and Methods. Average sizes of families in the SPS are compared using 2004-2006 enrollment records and other sources. Logistic models are used to identify explanatory variables for affiliation as a single-person family. Results. SPS families on average are composed of one member less in comparison with other sources. The logistic models indicate that the more recent the affiliation year the more probable is affiliation as a single-person family. Conclusions. The smaller number of family members implies an over-financing by the SPS. It is recommended to change the unit of financing to the individual to help the operational and financial sustainability of the SPS over the mid- to long-term.

Key words: Public Health Administration; Health Systems; Health Services Coverage; Social Security; Mexico.
T a creación del Sistema de Protección Social en SaLlud (SPSS) y de su respectiva Comisión Nacional (CNPSS) en 2003 tiene por objetivo la cobertura en salud del total de la población no derechohabiente de la seguridad social. Este sistema es comúnmente cono- cido como Seguro Popular de Salud (SPS) ya que este último es el brazo operativo del SPSS en la prestación de servicios médicos. ${ }^{1}$

Para el SPS, la unidad de protección social en salud es la familia conformada por los cónyuges (o concubina

(I) Unidad de Análisis Económico, Secretaría de Salud. México.

Fecha de recibido: 25 de marzo de 2009 • Fecha de aprobado: 4 de marzo de 2010

Solicitud de sobretiros: Cristina Gutiérrez Delgado. Unidad de Análisis Económico, Secretaría de Salud. Paseo de la Reforma 450 piso I4, col Juárez. 06600 México DF.

Correo electrónico: cgutierrezd@salud.gob.mx 
y concubinario), los hijos menores de 18 años y los padres mayores de 64 años que dependen económicamente de la familia y habitan en la misma vivienda. También se consideran los hijos solteros estudiantes de hasta 25 años o los discapacitados dependientes. ${ }^{2}$ Se considera como núcleo familiar a un individuo soltero mayor de 18 años y se le denomina familia unipersonal. Los gobiernos federal y estatales aportan financiamiento por familia para el funcionamiento del SPS definido con base en el 15\% de un salario mínimo vigente en el Distrito Federal el 1 de julio de 1997.² Tanto la definición de unidad de protección social como el porcentaje base de financiamiento se desarrollaron para tratar de homologarse con la definición de derechohabiente especificada por la Ley del Seguro Social, ${ }^{3}$ con la finalidad de, en un futuro, poder generar la portabilidad de derechos entre instituciones.

La afiliación al SPS es voluntaria y generalmente realizada por el titular de la familia. Al momento de afiliarse el titular presenta copia y original de documentos probatorios para cotejar información socioeconómica de su familia asentada en la Cédula de Características Socioeconómicas del Hogar (CECASOEH) ${ }^{4}$ La información se registra en el Sistema de Administración del Padrón Nacional de Beneficiarios (Padrón) bajo un identificador único por familia y por Ley se mantiene sin cambio durante los tres años de vigencia de la CECASOEH. ${ }^{4}$

La reforma a la Ley General de Salud (LGS) que crea al SPS, planea afiliar un máximo anual de 14.3\% del total de familias no cubiertas por la seguridad social durante el periodo 2004 - 2010. ${ }^{1}$ Las metas de afiliación para 2004, 2005 y 2006 se basan en la estimación de la población no asegurada en 2004..$^{-7}$

La Dirección General de Información en Salud (DGIS) de la Secretaría de Salud (SS) realizó en 2004 las estimaciones de la población y las familias susceptibles de afiliación al SPS, mediante la metodología siguiente: ${ }^{8}$ se establece un escenario donde los porcentajes tanto de población $(\beta)$ como de hogares con jefe no derechohabiente $(\alpha)$, en el plano nacional y por entidad federativa, se mantienen constantes de 2000 a 2004. Estos porcentajes se calcularon a partir de la muestra del Censo General de Población y Vivienda (CGPV). ${ }^{9}$ El porcentaje $(\alpha)$ se calcula directamente del CGPV 2000. Para calcular el porcentaje $(\beta)$ se divide a la población total en cuatro grupos: 1) derechohabientes con jefe de hogar derechohabiente, 2) no derechohabientes con jefe de hogar derechohabiente, 3) derechohabientes con jefe de hogar no derechohabiente, y 4) no derechohabientes con jefe de hogar no derechohabiente. Se emplea un algoritmo basado en las definiciones de derechohabiente de las instituciones de seguridad social con mayor cobertura en
México (Instituto Mexicano del Seguro Social e Instituto de Seguridad y Servicios Sociales de los Trabajadores del Estado) y se involucra a las variables parentesco, edad, derechohabiencia, discapacidad, asistencia a la escuela, estado conyugal y condición de actividad económica.

Para estimar el número de hogares en 2004 (ס) se toma el incremento promedio anual entre los CGPV 1990 y 2000, a nivel nacional y por entidad federativa, como constante en el periodo 2000-2004. Para estimar la población total en $2004(\varepsilon)$, se usa la proyección al 30 de junio de 2004 del Consejo Nacional de Población (CONAPO).

El número de familias susceptibles de afiliarse al SPS se obtiene multiplicando $(\alpha)$ por $(\delta)$, y el de la población no asegurada, multiplicando $(\beta)$ por $(\varepsilon)$. La cifra resultante fue 11.9 millones de familias. En noviembre de 2007 la DGIS actualizó las cifras con los resultados del CGPV 2005 y se obtuvieron 12.6 millones de familias. ${ }^{9}$ Cabe destacar que se maneja el término familia cuando realmente se trata de hogar.

El Instituto Nacional de Estadística, Geografía e Informática (INEGI) y el CONAPO definen hogar como la unidad formada por una o más personas, unidas o no por lazos de parentesco, que residen habitualmente en la misma vivienda y se sostienen de un gasto común para la alimentación. Jefe(a) de hogar es la persona reconocida como tal por los demás integrantes del hogar. Los hogares pueden ser familiares si por lo menos uno de los integrantes tiene relación de parentesco con el jefe del hogar o no familiares si ninguno de los integrantes tiene relación de parentesco con el jefe del hogar. ${ }^{10} \mathrm{La}$ definición de hogar es más general que la de familia definida por elSPS, ya que pueden existir varias familias en un hogar.

El objetivo de este artículo es comparar el promedio de integrantes por familia afiliada al SPS con el promedio de otras fuentes, e identificar las variables que mejor predicen la afiliación unipersonal.

\section{Material y métodos}

Las fuentes de información usadas son los padrones correspondientes al $4^{\circ}$ trimestre de $2004\left(2004 / 4^{\circ}\right), 2^{\circ}$ trimestre de $2005\left(2005 / 2^{\circ}\right)$ y $2^{\circ}$ trimestre de $2006\left(2006 / 2^{\circ}\right)$. Las otras fuentes son las Proyecciones de Población 2005-2050 11 y de Hogares 2000-2030 ${ }^{12}$ del CONAPO, Población no Asegurada y Núcleos Familiares (familias) sujetos de Afiliación al SPSS según la DGIS ${ }^{8}$ y Población Total y Hogares por Entidad Federativa del CGPV 2005. ${ }^{13}$ Stata 8.0 es el software seleccionado para realizar el análisis estadístico.

El padrón tiene una estructura muy similar a la CECASOEH en cuanto a las variables. Destaca que el 
formato del número de folio como identificador de familia cambió en el Padrón $2006 .{ }^{14}$ El padrón se actualiza trimestralmente, siguiendo los periodos de afiliación programados durante el año. ${ }^{4}$

En el Padrón 2004 $/ 4^{\circ}$ no existen registros de Chihuahua, Durango y Distrito Federal pues en estas entidades aún no estaba en operación el SPS. Por la misma razón, en el Padrón $2005 / 2^{\circ}$ no hay registros del Distrito Federal.

El método de análisis consta de cuatro etapas. En la primera se calcula el coeficiente de correlación de Pearson ${ }^{15}$ entre los promedios de integrantes por hogar o familia, en cada entidad federativa, de las diferentes fuentes de datos. El promedio de integrantes por hogar o familia se obtiene al dividir la población entre el número de hogares o familias. Las estimaciones de la DGIS se asumen constantes de 2004 a 2006, ya que se actualizaron hasta noviembre de 2007. El CGPV 2005 sólo se compara con el Padrón 2005/2 ${ }^{\circ}$.

En la segunda etapa se obtiene la distribución de los afiliados y de las familias unipersonales por edad y sexo para los tres padrones. También se obtiene la distribución de las familias unipersonales por edad y año de afiliación con los datos del Padrón 2006/2 , pues es el único que contiene la variable "año de afiliación".

En la tercera etapa se analizan las características de las familias unipersonales en los grupos de edad 18 a 25 años y 45 a 80 años en las variables "sexo", "trabaja", "estado civil" y "estudia" en los tres padrones; el significado de éstas se presenta en el cuadro I. También se obtiene el porcentaje de familias, en particular unipersonales, que se mantienen como tales del Padrón
$2004 / 4^{\circ}$ al $2005 / 2^{\circ}$ usando el número de folio como identificador entre las bases. No se puede realizar dicho seguimiento en el padrón $2006 / 2^{\circ}$ dado que cambió el número de folio.

En la cuarta etapa se aplica una regresión logística al Padrón $2006 / 2^{\circ}$ para identificar las variables más significativas en el hecho de que un titular se afilie unipersonalmente. Se elige este padrón dado que contiene la variable año de afiliación al SPS, los registros de todas las entidades federativas y el mayor número de afiliados, mejorando las inferencias estadísticas.

Ya que la manera en que se afilian los miembros de una familia depende del titular, se elige como observación de la regresión a éste último, pues es más razonable suponer que si se afilia en una familia unipersonal no afecta si lo hacen o no el resto de los titulares. Con este supuesto se cumple la hipótesis de independencia del modelo de regresión logística. 15,16

El modelo de regresión permite calcular la probabilidad de que un titular se afilie en una familia unipersonal mediante la vinculación de variables independientes incluidas en los padrones. Con la probabilidad obtenida se calcula la eficiencia predictiva de los modelos ajustados mediante las tasas de especificidad y sensibilidad ${ }^{15}$ que se definen como el porcentaje de titulares en familias no unipersonales que fueron clasificados correctamente y como el porcentaje de titulares en familias unipersonales clasificados correctamente.

El cuadro I presenta las variables del modelo de regresión logística. Las variables explicativas se seleccionaron a partir de las características de las familias unipersonales encontradas en la segunda y tercera etapas.

Cuadro I

\section{Características de familias afiliadas al Seguro Popular de Salud, México 2004-2006}

\begin{tabular}{|c|c|c|c|}
\hline Variable & Descripción & Tipo & Función en el modelo \\
\hline Unipersonal & $\begin{array}{l}\text { Si el titular está afiliado en una familia unipersonal, toma el valor de I; de } \\
0 \text { en otro caso }\end{array}$ & Binaria & $\begin{array}{l}\text { El logaritmo de su momio es la } \\
\text { variable respuesta }\end{array}$ \\
\hline Edad & Años de edad cumplidos al $2^{\circ}$ trimestre del 2006 & Continua & Variable explicativa \\
\hline Trabaja & ¿Trabaja? I: Sí; 2: No*; 3: Jubilado o pensionado & Cualitativa & Variable explicativa \\
\hline Estado civil & $\begin{array}{l}\text { Estado civil. I: Soltero(a)*; 2: Casado(a); 3: Divorciado(a); 4: Separado(a); 5: } \\
\text { Viudo(a); 6: Unión libre; 7: Otro tipo de unión. }\end{array}$ & Cualitativa & Variable explicativa \\
\hline Estudia & ¿Estudia? 0: No; I: Sí & Cualitativa & Variable explicativa \\
\hline Sexo & Sexo. 0: Hombre; I: Mujer. & Cualitativa & Variable explicativa \\
\hline Entidad federativa & Entidad federativa donde se ubica & Cualitativa & Variable explicativa \\
\hline Año de afiliación & Año en que se levantaron los datos de la CECASOEH. & Continua & Variable explicativa \\
\hline
\end{tabular}

Fuente: Elaboración propia a partir de las bases de datos del Padrón 2006/2º proporcionadas por la Dirección de Planeación y Administración del Padrón CNPSS, Secretaría de Salud. México 2008

* La variable indicadora correspondiente a esta categoría no fue incorporada en los modelos. En el caso de la variable. "entidad federativa", la variable indicadora correspondiente a Baja California no está considerada en el modelo 
Antes de realizar la regresión logística se eliminaron las observaciones con edad negativa o mayor de 106 años, y las que no tenían estatus asociado para las variables "sexo" y "trabaja" por considerarse inconsistencias. ${ }^{14} \mathrm{El} 11.23 \%$ de los afiliados no tenían estado civil asociado ${ }^{14}$ por lo que se decidió hacer dos supuestos para no descartarlos de la regresión: 1) los menores de 12 años son solteros ya que en la mayor parte de las entidades es la edad mínima para casarse y 2) para el resto de las edades se asigna un estado civil a los que carecen de él a partir de la distribución en cada edad de aquellos que sí lo tienen asociado. La asignación se realizó aleatoriamente con una distribución uniforme.

Las variables cualitativas que tenían más de dos categorías se recodificaron con una categoría menos a través de variables binarias, que toman el valor de 1 si el titular presentaba esa categoría y 0 en otro caso. ${ }^{15}$ Con base en la categoría que no fue considerada deben interpretarse los resultados de la variable cualitativa en cuestión. ${ }^{15}$ Con la recodificación hay 43 variables explicativas en total y se ajustan tres modelos: (a) con todos los titulares, ( $b$ ) con los titulares entre 18 y 25 años, y ( $c$ ) con los titulares entre 45 y 80 años de edad.

Las técnicas automatizadas de selección de variables forward, backward y stepwise no simplificaron los modelos, ya que la mayor parte de las 43 variables son altamente significativas. Por lo tanto, en los tres modelos se eliminaron manualmente algunas variables explicativas buscando modelos ajustados sencillos con una pérdida pequeña de eficiencia predictiva. Adicionalmente, se eliminó la variable binaria "trabaja" por tener un coeficiente de correlación lineal con "sexo" de -0.4377 en el modelo $b$, de -0.4521 en el modelo $c$ y de -0.4535 para el modelo $a$, lo que significa que la mayoría de las mujeres titulares de hogar reportaron no trabajar, por lo que resulta redundante la inclusión de la variable "trabaja".

Los resultados de los modelos de regresión logística se complementan con el cálculo de la razón de momios entre las variables para estimar si la presencia de una categoría en alguna de las variables tiene influencia sobre la probabilidad de que el titular se afilie unipersonalmente. Si la razón es cercana a cero no existe influencia de las variables analizadas.

\section{Resultados}

El cuadro II presenta los resultados de los tamaños promedios de hogares (INEGI, CONAPO) o de familias (DGIS, padrones), y los coeficientes de correlación lineal entre éstos. Se observa que las familias del SPS en promedio tienen un miembro menos respecto de otras fuentes. Existe una tendencia decreciente en el promedio de integrantes por familia afiliada y por hogar según todas las fuentes, pero la tendencia es más pronunciada en el SPS.

El coeficiente de correlación entre el Padrón 2004 / $4^{\circ}$ y la DGIS es el más alto, indicando que la estimación que realizó la DGIS de la población no asegurada en 2004 tiene una relación directa con los datos observados, pero la correlación disminuye para 2005 y 2006, pues no se actualizó la estimación 2004. Los coeficientes de correlación lineal positivos entre los padrones y el CONAPO indican que el tamaño de una familia afiliada sigue la tendencia decreciente proyectada del tamaño de un hogar. Sin embargo, el coeficiente para 2006 es el menor de todos, ya que los promedios tienden a cambiar en ritmos diferentes por entidad federativa.

El coeficiente de correlación entre el CGPV 2005 y el Padrón 2005 $/ 2^{\circ}$ indica que el tamaño de una familia afiliada y el tamaño observado de un hogar, en general, siguen la misma tendencia pero su relación es débil.

La figura 1(a) muestra que la mayoría de los afiliados son menores de 20 años, edad en la que existe un notable descenso en el número de afiliados. En contraste, las familias unipersonales aumentan significativamente alrededor de los 20 años según la figura 1(e).

La figura 1(e) también muestra que la distribución por edad de las familias unipersonales es similar en los tres padrones, por lo que los individuos que se afilian cada año unipersonalmente deben ser, en general, más jóvenes que los ya afiliados. Esto se confirma con la figura 1(f), que muestra que la distribución por edad al afiliarse se recorre a edades menores al aumentar el año de afiliación. La edad con mayor porcentaje de familias unipersonales para los tres padrones es 20 años como consecuencia de la similitud entre las tres distribuciones por edad.

Las figuras 1(b) a 1(d) exhiben que hay más mujeres afiliadas unipersonalmente que hombres, hecho que se repite para casi todas las edades. También existen familias unipersonales menores de 18 años en todos los padrones. En 2006 se afiliaron más familias unipersonales menores de 18 años con respecto a 2004 y 2005 como se muestra en la figura 1(f).

El cuadro III presenta los resultados de condición laboral, estado civil y / o condición escolar para los grupos de edad 18 a 25 años y 45 a 80 años debido a que contienen alrededor del 28 y $45 \%$ de familias unipersonales. El número de mujeres afiliadas unipersonalmente es mayor que el de los hombres y tiene una tendencia creciente, pues de $2004 / 4^{\circ}$ a $2006 / 2^{\circ}$ aumentó de 64.4 a $65.4 \%$.

Para las familias unipersonales entre 18 y 25 años en el periodo $2005 / 2^{\circ}-2006 / 2^{\circ}$, el porcentaje de individuos solteros decreció, mientras que los de casados y en unión libre crecieron. El porcentaje de individuos entre 18 y 25 
Cuadro II

COMPARACIÓN DE PROMEDIOS DE INTEGRANTES POR HOGARES O FAMILIAS afiliados al Seguro Popular de Salud, México 2004-2006

\begin{tabular}{|c|c|c|c|c|c|c|c|c|}
\hline \multirow{2}{*}{ Entidad Federativa } & \multicolumn{3}{|c|}{2004} & \multicolumn{3}{|c|}{2005} & \multicolumn{2}{|c|}{2006} \\
\hline & SPSS* & $D G I S^{\ddagger}$ & CONAPO§ & SPSS & $C P V^{\#}$ & CONAPO & SPSS & CONAPO \\
\hline Aguascalientes & 3.56 & 5.50 & 4.46 & 3.34 & 4.28 & 4.38 & 3.51 & 4.31 \\
\hline Baja California & 2.96 & 4.79 & 3.87 & 3.02 & 4.08 & 3.82 & 2.70 & 3.77 \\
\hline Baja California Sur & 3.16 & 4.84 & 3.86 & 3.18 & 3.87 & 3.80 & 3.26 & 3.75 \\
\hline Campeche & 3.01 & 4.88 & 4.18 & 2.82 & 4.05 & 4.12 & 3.21 & 4.05 \\
\hline Coahuila & 3.06 & 5.11 & 3.99 & 3.06 & 3.98 & 3.94 & 3.22 & 3.88 \\
\hline Colima & 2.75 & 4.48 & 3.80 & 2.74 & 3.86 & 3.74 & 3.74 & 3.68 \\
\hline Chiapas & 3.39 & 5.07 & 4.51 & 3.33 & 4.64 & 4.43 & 3.35 & 4.35 \\
\hline Chihuahua & - & - & - & 3.42 & 3.94 & 3.80 & 3.23 & 3.74 \\
\hline Distrito Federal & - & - & - & - & - & - & 3.25 & 3.64 \\
\hline Durango & - & - & - & 3.44 & 4.19 & 4.16 & 3.19 & 4.09 \\
\hline Guanajuato & 3.40 & 5.13 & 4.60 & 3.34 & 4.43 & 4.52 & 3.12 & 4.44 \\
\hline Guerrero & 3.13 & 4.74 & 4.36 & 3.25 & 4.36 & 4.29 & 3.35 & 4.22 \\
\hline Hidalgo & 3.48 & 4.61 & 4.32 & 3.49 & 4.17 & 4.25 & 3.38 & 4.18 \\
\hline Jalisco & 3.58 & 4.81 & 4.17 & 3.56 & 4.23 & 4.11 & 3.46 & 4.05 \\
\hline Estado de México & 3.92 & 5.13 & 4.10 & 3.53 & 4.35 & 4.03 & 3.64 & 3.97 \\
\hline Michoacán & 4.01 & 4.62 & 4.34 & 4.02 & 4.23 & 4.27 & 3.56 & 4.19 \\
\hline Morelos & 3.36 & 4.63 & 3.90 & 3.34 & 4.06 & 3.85 & 3.14 & 3.79 \\
\hline Nayarit & 3.03 & 4.56 & 3.98 & 3.04 & 3.89 & 3.92 & 3.10 & 3.86 \\
\hline Nuevo León & 3.59 & 5.02 & 4.03 & 3.58 & 4.07 & 3.97 & 3.59 & 3.92 \\
\hline Oaxaca & 2.96 & 4.67 & 4.46 & 2.89 & 4.26 & 4.38 & 2.77 & 4.31 \\
\hline Puebla & 4.01 & 4.98 & 4.40 & 4.01 & 4.40 & 4.33 & 3.71 & 4.26 \\
\hline Querétaro & 4.40 & 5.32 & 4.28 & 4.39 & 4.32 & 4.21 & 4.21 & 4.13 \\
\hline Quintana Roo & 3.81 & 4.98 & 3.87 & 3.56 & 4.41 & 3.81 & 3.60 & 3.76 \\
\hline San Luis Potosí & 3.89 & 4.83 & 4.47 & 3.69 & 4.24 & 4.39 & 3.26 & 4.32 \\
\hline Sinaloa & 3.64 & 5.12 & 4.14 & 3.70 & 4.13 & 4.08 & 3.60 & 4.01 \\
\hline Sonora & 3.22 & 4.97 & 4.00 & 3.10 & 3.95 & 3.94 & 2.92 & 3.89 \\
\hline Tabasco & 3.12 & 4.87 & 4.26 & 2.96 & 4.13 & 4.18 & 3.07 & 4.10 \\
\hline Tamaulipas & 3.17 & 4.71 & 3.83 & 3.14 & 3.88 & 3.78 & 3.13 & 3.73 \\
\hline Tlaxcala & 3.73 & 5.27 & 4.52 & 3.69 & 4.43 & 4.44 & 3.32 & 4.36 \\
\hline Veracruz & 3.42 & 4.56 & 4.05 & 3.38 & 3.95 & 3.99 & 3.11 & 3.92 \\
\hline Yucatán & 4.02 & 4.98 & 4.18 & 4.06 & 4.11 & 4.12 & 3.41 & 4.06 \\
\hline Zacatecas & 3.15 & 4.49 & 4.32 & 3.63 & 4.14 & 4.25 & 3.29 & 4.18 \\
\hline Nacional & 3.40 & 4.84 & 4.14 & 3.32 & 4.17 & 4.07 & 3.28 & 4.01 \\
\hline & & & & & & SPSS & & \\
\hline & & & 200 & & & & & \\
\hline DGIS & & & & & & & & \\
\hline CONAPO & & & & & & & & \\
\hline INEGI & & & & & & & & \\
\hline
\end{tabular}

Fuente: Cálculos de los autores a partir de:

* Las bases de datos de los afiliados al SPS proporcionadas por la Dirección de Planeación y Administración del Padrón de la CNPSS, Secretaría de Salud, 2008

‡ Población no asegurada y Núcleos Familiares sujetos de afiliación al SPSS en 2004 estimada por la DGIS de la Secretaría de Salud

$\S$ Proyecciones de Hogares 2000 - 2030 y Proyecciones de la Población 2005 - 2050, ambas del CONAPO

\# CPV 2005, INEGI

No se muestran los promedios de las entidades que no tienen registros en los padrones 


\section{(a) Afiliados}

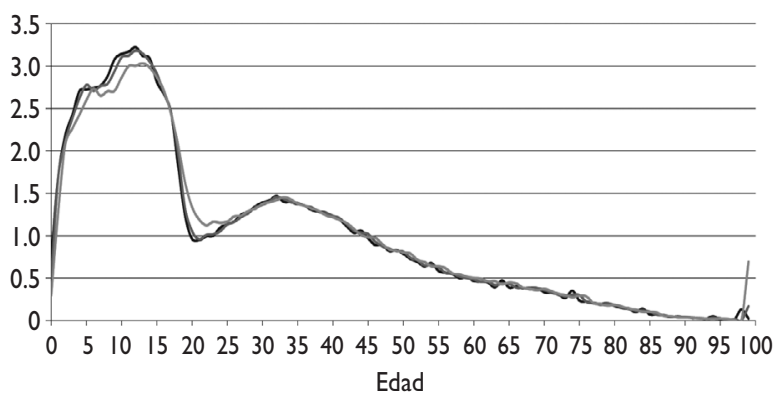

Padrón

$$
\begin{aligned}
& \text { 2004/4o. } \\
& \text { 2005/2o. } \\
& \text { 2006/2o. }
\end{aligned}
$$

(b) Familias unipersonales-padrón 2004/4

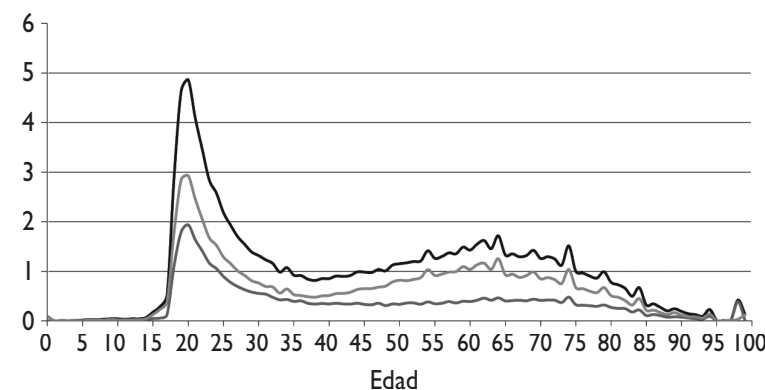

Total

Hombres

Mujeres

(d) Familias unipersonales-padrón 2005/2

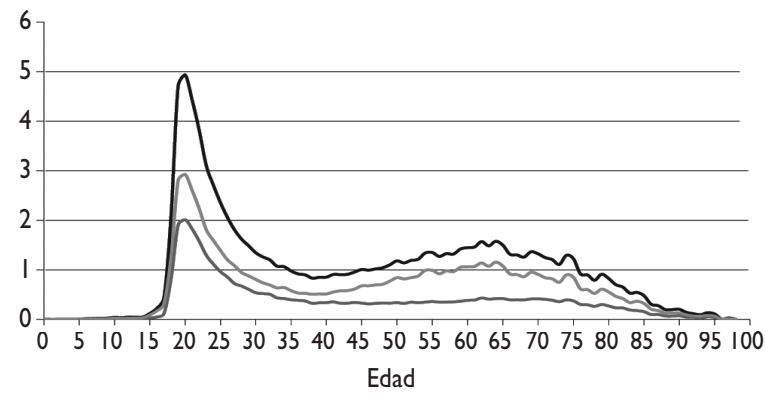

Total

Hombres

Mujeres (d) Familias unipersonales-padrón 2006/2

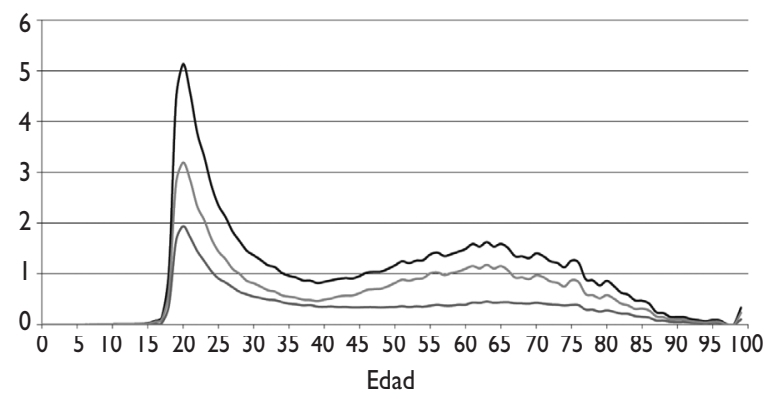

Total

Hombres

Mujeres

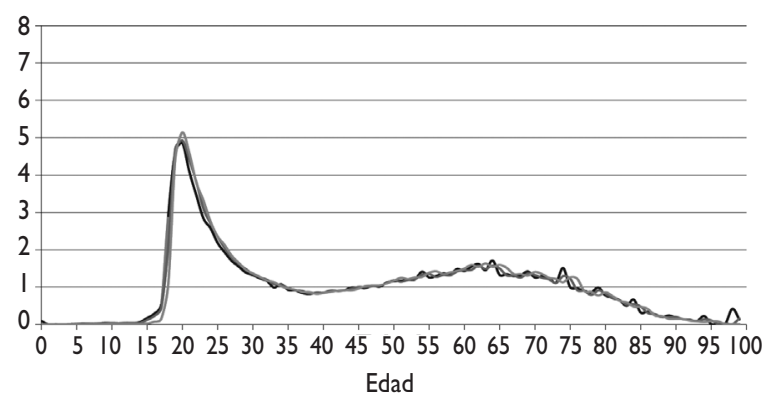

Padrón

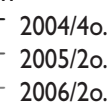

(f) Distribución de familias unipersonales por edad al momento de afiliación*

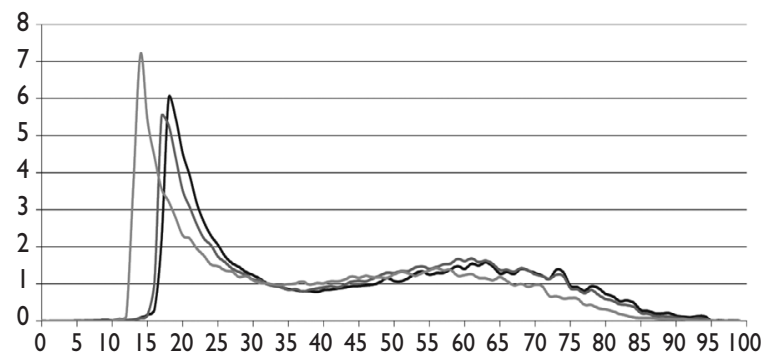
Edad

Año de afiliación

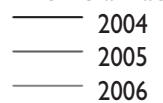

Fuente: Cálculos de los autores partir de las bases de datos de los afiliados al SPS proporcionadas por la Dirección de Planeación y Administración del Padrón - CNPSS, Secretaría de Salud. México 2008

Los porcentajes son respecto del total de familias unipersonales

Los datos graficados en (e) pertenecen al Padrón $2006 / 2^{\circ}$, ya que es el único que contiene la variable "año de afiliación". La edad fue actualizada al año de afiliación de la persona al SPS

Figura I. Estructura por edad. Familias unipersonales afiliadas al Seguro Popular de Salud, México 2004-2006 
Cuadro III

Características de familias unipersonales afiliadas al Seguro Popular de Salud, México 2004-2006

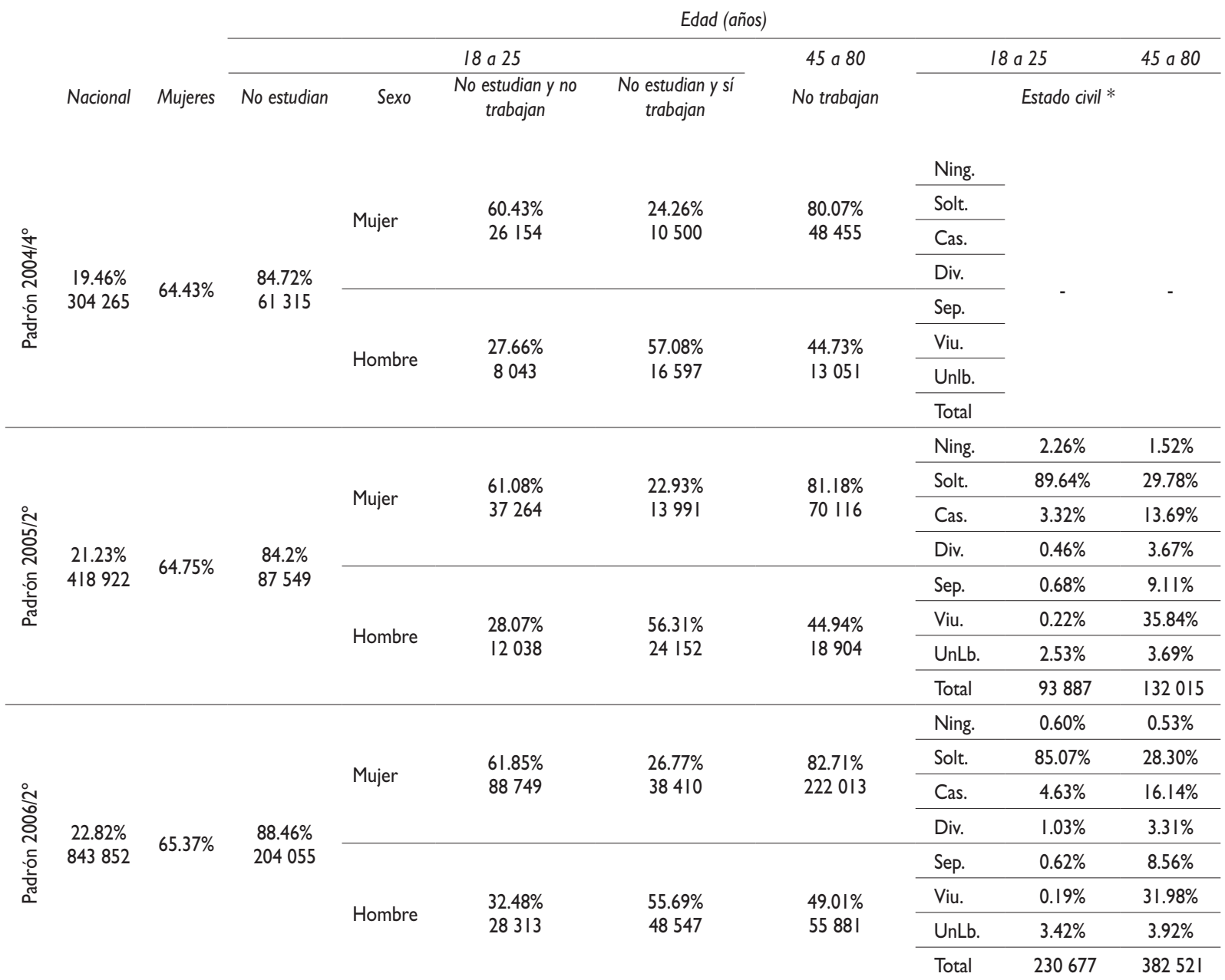

Fuente: Cálculos de los autores a partir de las bases de datos de los afiliados al SPS proporcionadas por la Dirección de Planeación y Administración del Padrón - CNPSS. México 2008

El número de familias unipersonales entre 18 y 25 años de edad es: $83733\left(2004 / 4^{\circ}\right)$, II $17672\left(2005 / 2^{\circ}\right)$ y $23068 \mid\left(2006 / 2^{\circ}\right)$

El número de familias unipersonales entre 45 y 80 años de edad es: I35 $185\left(2004 / 4^{\circ}\right), 192075\left(2005 / 2^{\circ}\right)$ y $407170\left(2006 / 2^{\circ}\right)$

* En el Padrón $2004 / 4^{\circ}$ no estuvo disponible la variable estado civil. Significado de las abreviaciones: Ning.- ninguno, Solt.- soltero, Cas.- casado, Div.- divorciado, Sep.- separado,Viu.- viudo y Unlb.- unión libre

años de edad que no estudian y no trabajan se incrementó, y sólo el porcentaje de hombres que no estudian y sí trabajan decreció, tal como se esperaba de la definición de unidad de protección social en salud. Sin embargo, un porcentaje creciente de dichas familias se encuentra en unión con otra persona, lo mismo ocurre en el grupo de edad 45 a 80 años, lo que genera una inconsistencia en el registro del estado civil en los padrones. En las familias unipersonales entre 45 y 80 años, el porcentaje de personas que no trabajan es creciente, principalmente en mujeres.
En lo que respecta a la permanencia de las familias, 1474655 familias afiliadas en $2004 / 4^{\circ}$ se mantienen al $2005 / 2^{\circ}(94.31 \%)$ y 268697 familias unipersonales afiliadas en $2004 / 4^{\circ}$ permanecieron así al $2005 / 2^{\circ}(88.31 \%)$. Un total de 51653 familias dejaron de ser unipersonales para $2005 / 2^{\circ}$, de las cuales 26800 ya no aparecen en el Padrón $2005 / 2^{\circ}$, y 24853 familias incrementaron su número de integrantes. Destaca que el porcentaje general de reafiliación es menor al reportado por la CNPSS $(96.5 \%) .{ }^{5}$ Tal discrepancia puede deberse a la diferente fecha de corte de los padrones. 
En la regresión logística, una vez eliminadas las observaciones inconsistentes y asignado el estado civil, quedaron 12075023 personas y 3696013 titulares de familia, por lo que sólo se perdieron 2630 titulares (0.07\% de los titulares totales).

El cuadro IV muestra los modelos logísticos ajustados. En el modelo a si un(a) titular viudo(a) se va a afiliar, la probabilidad de que lo haga unipersonalmente se incrementa 30\% respecto de si fuera soltero. Además, conforme el año de afiliación es más reciente, la probabilidad de afiliarse unipersonalmente se incrementa $48 \%$.
El resto de las variables disminuyen la probabilidad de afiliarse unipersonalmente.

En el modelo $b$ si un(a) titular estudia, entonces la probabilidad de afiliarse unipersonalmente se incrementa $117 \%$. Conforme el año de afiliación es más reciente, aumenta $42 \%$ la probabilidad de afiliarse unipersonalmente. Las otras variables disminuyen la probabilidad de afiliarse unipersonalmente.

Para el modelo $c$, por cada año que aumenta la edad del titular o conforme el año de afiliación es más reciente, la probabilidad de afiliarse unipersonalmente aumenta 7

\section{Modelos ajustados para explicar la afiliación de familias unipersonales al Seguro Popular de Salud, México 2004-2006}

\begin{tabular}{|c|c|c|c|c|c|c|}
\hline & Variable & $R M$ & IC $95 \%$ & Valor $p$ & $\begin{array}{c}\text { Sensibilidad } \\
\%\end{array}$ & $\begin{array}{c}\text { Especificidad } \\
\%\end{array}$ \\
\hline \multirow{9}{*}{$\begin{array}{l}\stackrel{0}{0} \\
\stackrel{\circ}{0} \\
\frac{0}{2} \\
\Sigma\end{array}$} & Sexo & 0.2866 & $(0.2846,0.2887)$ & $<0.0001$ & \multirow{9}{*}{63.76} & \multirow{9}{*}{90.59} \\
\hline & \multicolumn{4}{|l|}{ Estado civil } & & \\
\hline & Casado(a) & 0.0480 & $(0.0476,0.0484)$ & $<0.0001$ & & \\
\hline & Divorciado(a) & 0.5208 & $(0.5|05,0.53| 2)$ & $<0.0001$ & & \\
\hline & Separado(a) & 0.2225 & $(0.2198,0.2252)$ & $<0.0001$ & & \\
\hline & Viudo(a) & 1.3075 & $(1.2948,1.3203)$ & $<0.0001$ & & \\
\hline & Unión libre & 0.0531 & $(0.0525,0.0537)$ & $<0.0001$ & & \\
\hline & Otro tipo de unión & 0.0779 & $(0.0730,0.083 \mathrm{I})$ & $<0.0001$ & & \\
\hline & Año de afiliación & 1.4824 & $(\mathrm{I} .4770, \mathrm{I} .4879)$ & $<0.0001$ & & \\
\hline \multirow{11}{*}{$\begin{array}{l}\text { 을 } \\
\frac{0}{\mathrm{~d}} \\
\frac{\mathrm{O}}{\Sigma}\end{array}$} & Edad & 0.8536 & $(0.8504,0.8567)$ & $<0.0001$ & \multirow{11}{*}{86.22} & \multirow{11}{*}{83.26} \\
\hline & Estado civil & & & & & \\
\hline & Casado(a) & 0.0352 & $(0.0345,0.0359)$ & $<0.0001$ & & \\
\hline & Divorciado(a) & 0.3902 & $(0.3667,0.4153)$ & $<0.0001$ & & \\
\hline & Separado(a) & 0.0612 & $(0.0580,0.0646)$ & $<0.0001$ & & \\
\hline & Viudo(a) & 0.1063 & $(0.0957,0.118 \mathrm{I})$ & $<0.0001$ & & \\
\hline & Unión libre & 0.0324 & $(0.0317,0.0331)$ & $<0.0001$ & & \\
\hline & Otro tipo de unión & 0.0489 & $(0.0424,0.0565)$ & $<0.0001$ & & \\
\hline & Estudia & 2.1681 & $(2.1019,2.2363)$ & $<0.0001$ & & \\
\hline & Sexo & 0.3052 & $(0.2999,0.3107)$ & $<0.0001$ & & \\
\hline & Año de afiliación & 1.4159 & $(\mathrm{I} .4027, \mathrm{I} .4293)$ & $<0.0001$ & & \\
\hline \multirow{10}{*}{$\begin{array}{l}u \\
\frac{0}{0} \\
\frac{0}{0} \\
\sum\end{array}$} & Edad & 1.0698 & $(1.0692,1.0703)$ & $<0.0001$ & \multirow{10}{*}{63.35} & \multirow{10}{*}{89.74} \\
\hline & Estado civil & & & & & \\
\hline & Casado(a) & 0.0657 & $(0.0649,0.0666)$ & $<0.0001$ & & \\
\hline & Divorciado(a) & 0.8945 & $(0.8670,0.9228)$ & $<0.0001$ & & \\
\hline & Separado(a) & 0.4845 & $(0.4758,0.4934)$ & $<0.0001$ & & \\
\hline & Viudo(a) & 0.8368 & $(0.8244,0.8494)$ & $<0.0001$ & & \\
\hline & Unión libre & 0.1016 & $(0.0996,0.1036)$ & $<0.0001$ & & \\
\hline & Otro tipo de unión & 0.1362 & $(0.1227,0.1513)$ & $<0.0001$ & & \\
\hline & Sexo & 0.6113 & $(0.6046,0.6180)$ & $<0.0001$ & & \\
\hline & Año de afiliación & 1.5922 & $(1.5833,1.6012)$ & $<0.0001$ & & \\
\hline
\end{tabular}

Fuente: Cálculos de los autores a partir de la base de datos del Padrón 2006/2 ${ }^{\circ}$ proporcionada por la Dirección de Planeación y Administración del Padrón CNPSS, Secretaría de Salud. México 2008

RM: Razón de momios

IC $95 \%$ : Intervalo de confianza del $95 \%$ 
y 59\%, respectivamente. El resto de las variables disminuye la probabilidad de afiliarse unipersonalmente.

\section{Discusión}

El resultado más importante del análisis es el hecho de que en promedio las familias afiliadas al SPS presentan un integrante menos respecto al originalmente considerado. En particular resalta la tendencia creciente en el porcentaje de familias unipersonales en el ámbito nacional de 19.5 a $22.8 \%$ en tres años. Estos resultados son contrarios a lo esperado ya que la población objetivo del SPS se caracteriza por tener bajos ingresos y un menor grado de escolaridad, lo cual se relaciona con familias de mayor número de integrantes. ${ }^{9}$ Los resultados obtenidos tienen implicaciones importantes tanto a nivel operativo como a nivel de financiamiento para el funcionamiento sustentable del SPS en el mediano y largo plazo. Financieramente, la asignación por persona para cubrir el Catálogo de Servicios Esenciales de Salud provisto por el SPS ascendió a \$1 617 en $2006 .{ }^{17}$ Considerando la diferencia existente en el tamaño de la familia promedio afiliada en comparación con el tamaño original estimado por la DGIS, el SPS transfirió a los estados operarios del SPS aproximadamente $40 \%$ más de los recursos financieros realmente necesarios en el periodo 2004 a 2006. Además, el tamaño de familia se considera para la planificación de nueva infraestructura y la definición de redes de atención, por lo que un tamaño menor implica una sobre-estimación en estos conceptos.

En términos técnicos, el tamaño promedio observado de las familias afiliadas al SPS y la diferencia entre las definiciones de familia y hogar indican que no existe un método robusto que permita estimar de forma confiable el número de familias susceptibles de afiliación. Por ejemplo, en Baja California y Tabasco, la población afiliada en 2007 superó la población potencial de afiliación inicial, pues los cálculos de la DGIS están subestimados. ${ }^{10}$

La regresión logística arroja que la variable año de afiliación tiene el mayor poder predictivo para afiliarse unipersonalmente. Dicho resultado indica que cuanto más reciente sea el año de afiliación mayor es la probabilidad de ser afiliado como familia unipersonal. La implicación de este hallazgo es que la afiliación unipersonal depende de un variable ajena a las variables socioeconómicas de la familia. Este resultado pudiera vincularse con el proceso de negociación anual para fijar la meta de afiliación por estado y al hecho de que por cada peso que el estado invierte por familia afiliada, el estado recibe tres pesos por parte del gobierno federal a través de la CNPSS. ${ }^{2}$ Desafortunadamente la informa- ción disponible en los padrones no permite realizar un análisis para vincular los incentivos financieros con la probabilidad de afiliación unipersonal. Sin embargo, en una futura investigación pudiera realizarse un análisis de flujos financieros por estado y su posible relación con la afiliación.

Cabe resaltar que el número tan bajo de miembros por familia en estados como Oaxaca o Baja California puede deberse a un fenómeno de migración. Sin embargo, otra de las limitaciones de este análisis es la carencia de datos en los padrones que permitan vincular el tipo de trabajo, tiempo de residencia y el lugar de origen con el tamaño de la familia para determinar la influencia del fenómeno migratorio en la afiliación unipersonal.

De los resultados obtenidos y sus implicaciones se recomienda replantear que el financiamiento gubernamental se realice por persona manteniendo la afiliación por familia. Este cambio representaría definir como base de contribución un porcentaje que fluctuaría entre el 3 y el 3.6\% del salario mínimo establecido en la LGS por persona, considerando las estimaciones de la DGIS o del INEGI, respectivamente. ${ }^{8,13}$ Esta nueva base de financiamiento tiene dos ventajas. Por un lado ayuda a optimizar la asignación financiera, la planeación de infraestructura y la generación de redes de atención. Por otro lado, permite en el mediano plazo mejorar la estimación del tamaño de familia afiliada al SPS que se esperaría más cercana a la estimación basada en otras fuentes. Adoptar la unidad de financiamiento por persona ayudará a lograr el sostenimiento del SPS en el mediano y largo plazo, sentando precedente para apoyar la optimización en la asignación recursos para la salud en el contexto de la Ley del Seguro Social y, en el largo plazo, en el contexto de la portabilidad de derechos en salud.

\section{Agradecimientos}

Agradecemos a la CNPSS por facilitar las bases de datos de los padrones. También agradecemos los valiosos comentarios de Mariana Barraza, Livia Villa y de los revisores anónimos que enriquecieron este trabajo. Cristina Gutiérrez agradece el financiamiento del CONACyT.

\section{Referencias}

I. Secretaría de Salud. Sistema de Protección Social en Salud. Elementos conceptuales, financieros y operativos. Eduardo González Pier, Mariana Barraza Lloréns, Cristina Gutiérrez Delgado,Armando Vargas Palacios (coord). $2^{\mathrm{a}}$ ed. México: FCE, Secretaría de Salud, 2006.

2. Presidencia de la República. Reforma a la Ley General de Salud. Diario Oficial de la Federación, I5 de mayo 2003.

3. Cámara de Diputados, Ley del Seguro Social, 200. 
4. Secretaría de Salud. Lineamientos para la Afiliación, Operación, Integración del Padrón Nacional de Beneficiarios y Determinación de la Cuota Familiar del Sistema de Protección Social en Salud. Diario Oficial de la Federación, I5 de abril 2005.

5. Comisión Nacional de Protección Social en Salud. Indicadores de Resultados, Resultado del Primer Semestre 2004. México: Secretaría de Salud, 2005. [Consultado: 2008 abril I]. Disponible en: http://www. salud.gob.mx/transparencia/informes/segpopular/Informe_segundo_ semestre2005.pdf.

6. Comisión Nacional de Protección Social en Salud. Indicadores de Resultados, Resultado del Segundo Semestre 2005. México: Secretaría de Salud, 2006.

7. Comisión Nacional de Protección Social en Salud. Indicadores de Resultados, Segundo Semestre 2006. México: Secretaría de Salud, 2007. 8. Suárez Valdés Ayala A. et al. Síntesis Ejecutiva: Población no asegurada y Núcleos Familiares sujetos de afiliación al Sistema de Protección Social en Salud. México: Secretaría de Salud, 2004. [Consultado: 2008 abril I]. Disponible en: http://sinais.salud.gob.mx/poblacion/ PobNoAseguradyNucleosFam.pdf.

9. Comisión Nacional de Protección Social en Salud. Informe de Resultados 2007. México: Secretaría de Salud, 2008.

10. Instituto Nacional de Estadística, Geografía e Informática. Glosario del Censo General de Población y Vivienda 2000. México: Instituto Nacional de Estadística, Geografía e Informática, 2005. [Consultado: 2008 marzo 4]. Disponible en: http://www.inegi.gob.mx/est/contenidos/espanol/rutinas/ glogen $/$ default.aspx?t=CP\&s=est\&c=10249

I I. Partida V. Proyecciones de la Población de México 2005-2050. México: Consejo Nacional de Población, 2006. [Consultado: 2008 marzo 4].

Disponible en: http://www.conapo.gob.mx/00cifras/proy/Proy05-50.pdf. 12. Proyecciones de Hogares 2000-2030. México: CONAPO, 2000. [Consultado 2008 mayo I]. Disponible en: http://www.conapo.gob. $\mathrm{mx} / 00$ cifras $/ 0 \mathrm{l}$.htm

13. Conteo de Población y Vivienda 2005 - Población Total y Número de Hogares por Entidad Federativa. México: INEGI, 2005. [Consultado 2008 mayo I]. Disponible en: http://www.inegi.gob.mx/inegi/default. aspx?s=est\&c $=10215$.

14. Pérez-Salvador J.Análisis de la Consistencia del Padrón de Afiliados del Sistema de Protección Social en Salud de México. Documento de trabajo 03/2008, 2008.

15. Ramírez-Velásquez A.Algunas aplicaciones de los modelos de regresión logística. (Tesis). México: Universidad Nacional Autónoma de México, 2005.

16. Agresti A. Categorical Data Analysis. New York: John Wiley \& Sons, 1990. 17. Comisión Nacional de Protección Social en Salud, Catálogo de Servicios Esenciales de Salud 2006. México: CNPSS, 2006. 